\title{
Unique surface-state connection between Weyl and nodal ring fermions in ferromagnetic material $\mathrm{Cs}_{2} \mathrm{MoCl}_{6}$
}

\author{
Tiantian Zhang $\odot,{ }^{1,2, *}$ Daisuke Hara, ${ }^{1}$ and Shuichi Murakami ${ }^{1,2}$ \\ ${ }^{1}$ Department of Physics, Tokyo Institute of Technology, Ookayama, Meguro-ku, Tokyo 152-8551, Japan \\ ${ }^{2}$ Tokodai Institute for Element Strategy, Tokyo Institute of Technology, Nagatsuta, Midori-ku, \\ Yokohama, Kanagawa 226-8503, Japan
}

(Received 1 September 2021; accepted 16 November 2021; published 9 December 2021)

\begin{abstract}
For topological materials with a coexistence of Weyl nodes and nodal rings, their unique surface-state configuration and connection still need to be studied and discussed. In this Letter, we predict a ferromagnetic (FM) material, $\mathrm{Cs}_{2} \mathrm{MoCl}_{6}$, with a coexistence of Weyl and nodal ring fermions in its spinful FM electronic band structure, which is unusual since FM materials are very rare in nature and nodal ring band crossings will usually open a gap when spin-orbit coupling is taken into consideration. We find that the surface states of $\mathrm{Cs}_{2} \mathrm{MoCl}_{6}$ show different properties along different directions, i.e., the surface states are in a drumhead shape showing the nodal ring property on the (001) surface and in a helicoid shape showing the Weyl property on the (010) surface. Interestingly, both the drumhead surface states and the helicoid surface states will cross the projected points of the Weyl and nodal ring along different directions. In particular, helicoid surface states on the (010) surface will meet the nodal ring tangentially, with their shapes changing abruptly as a function of the energy. We implement both first-principles calculation and an analytical model to understand the unique surface-state connection for systems with a coexistence of Weyl nodes and nodal rings (or nodal lines). This result is universal and irrespective of the presence or absence of time-reversal symmetry $(\mathcal{T})$.
\end{abstract}

DOI: 10.1103/PhysRevResearch.3.L042037

Introduction. In the recent years, topological magnetic materials [1-17] have drawn much attention due to their exotic properties, e.g., topological axion insulator states [18-20], an intrinsic quantum anomalous Hall effect without external magnetic fields $[6,7,9,11-13]$, and the display of magnetism, topology, spin dynamics, and quantum transport. However, experimentally synthesized and verified magnetic topological materials are very limited, especially for magnetic topological semimetals $[8,10-16]$. Furthermore, compared to the bulk topology, the properties of topological surface states such as the configuration and connection are not so well understood in some systems. In this Letter, we propose a "high-quality" ferromagnetic (FM) material $\mathrm{Cs}_{2} \mathrm{MoCl}_{6}$ [21] with space group $F m \overline{3} m$ that has both Weyl and nodal ring fermions in its spinful electronic band structure. We find a unique surfacestate connection between the Weyl and nodal ring fermions along different surface directions by both density functional theory (DFT) and model analysis due to an intriguing interplay between drumhead surface states (DSSs) from the nodal rings and helicoid surface states (HSSs), i.e., Fermiarc surface states, from the Weyl nodes. We show that the DSSs always go across the Weyl nodes, when the projection of the Weyl nodes is inside that of the nodal ring. We also

\footnotetext{
*zhang.t.ac@m.titech.ac.jp

Published by the American Physical Society under the terms of the Creative Commons Attribution 4.0 International license. Further distribution of this work must maintain attribution to the author(s) and the published article's title, journal citation, and DOI.
}

show that how the HSSs connect the projection of the nodal ring and the curvature of HSSs depends sensitively on the energy. Such a unique surface-state connection also appears in several previous studies, yet lacks discussion [6,22-25]. Our results show that the connection pattern of the surface states is surface dependent for systems with both Weyl and nodal ring fermions, and it is universal for systems with or without considering spin-orbit coupling (SOC) and time-reversal $(\mathcal{T})$ symmetry.

Crystal and magnetic structure for $\mathrm{Cs}_{2} \mathrm{MoCl}_{6}$. $\mathrm{Cs}_{2} \mathrm{MoCl}_{6}$ belongs to space group No. 225, and the lattice constant we use in this Letter is $a=10.57 \AA$ after a relaxation on the atomic positions, which is slightly different from the experimental data $10.21 \AA$ at room temperature [21]. For most of the compounds having a similar crystal structure as $\mathrm{Cs}_{2} \mathrm{MoCl}_{6}$ (e.g., replacing $\mathrm{Cs}, \mathrm{Mo}$, and $\mathrm{Cl}$ by other alkali metal elements, transition metal elements, and halogen elements, respectively), their magnetic properties have yet to be explored by experiments except for $\mathrm{Cs}_{2} \mathrm{CoF}_{6}$ and $\mathrm{Rb}_{2} \mathrm{CoF}_{6}$, which have a FM phase at low temperature [26], and $\mathrm{Cs}_{2} \mathrm{MoCl}_{6}$ is also among the ones lacking exploration.

In order to explore the magnetic structure of the ground state in $\mathrm{Cs}_{2} \mathrm{MoCl}_{6}$, we calculate the total energy of the systems with three different magnetic structures for No. 225 by DFT, which are shown in Figs. 1(c)-1(e). The total energy for the FM phase in Figs. 1(c) and 1(d) is about $20 \mathrm{meV}$ lower than the antiferromagnetic phase in Fig. 1(e) per unit cell, and the FM phase with magnetic order on the (001) surface [Fig. 1(c)] will have a smaller total energy of about $1 \mathrm{meV}$ per unit cell than that along the [111] direction [Fig. 1(d)]. Thus, we will choose the one with a magnetic moment 
(a)

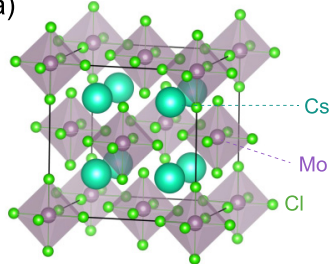

(b)
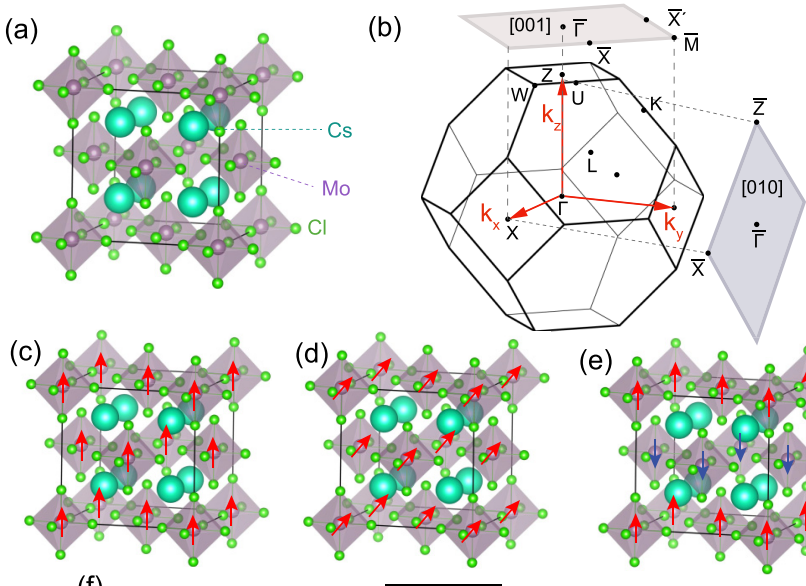

(d) $270 \%$ (e)
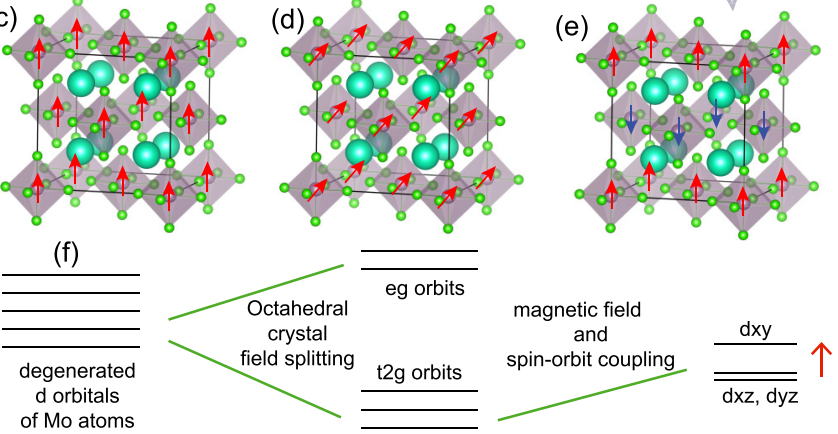

FIG. 1. Crystal structure, Brillouin zone (BZ), and splitting of $d$ orbitals of $\mathrm{Cs}_{2} \mathrm{MoCl}_{6}$. (a) and (b) are the crystal structure and (surface) Brillouin zone of $\mathrm{Cs}_{2} \mathrm{MoCl}_{6}$. (c)-(e) are three different types of magnetic structures of $\mathrm{Cs}_{2} \mathrm{MoCl}_{6}$ with a ferromagnetic phase along the [001] direction, [111] direction, and antiferromagnetic phase, respectively. (f) shows the splitting of $d$ orbitals under different fields.

along the highest-symmetry direction, i.e., the [001] direction, to explore the topological properties in $\mathrm{Cs}_{2} \mathrm{MoCl}_{6}$. In the following, we will introduce the topology of the electronic band structure of $\mathrm{Cs}_{2} \mathrm{MoCl}_{6}$ both in the nonmagnetic (NM) phase and in the FM phase separately.

Band structure of $\mathrm{Cs}_{2} \mathrm{MoCl}_{6}$. In the spinless $\mathrm{NM}$ electronic band structure, $d$ orbitals from Mo will split into $e_{g}$ and $t_{2 g}$ orbitals due to the octahedral crystal field environment, and the splitting is very huge due to the large $p$ - $d$ hybridization of Mo and Cl, as shown in Figs. 1(f) and 2(a). Thus, we only need to study the $t_{2 g}$ orbitals located near the Fermi energy, and they exhibit nontrivial topologies, i.e., nodal chains formed by the two lowest $t_{2 g}$ orbitals crossing the Fermi level. Due to the existence of mirror symmetries,
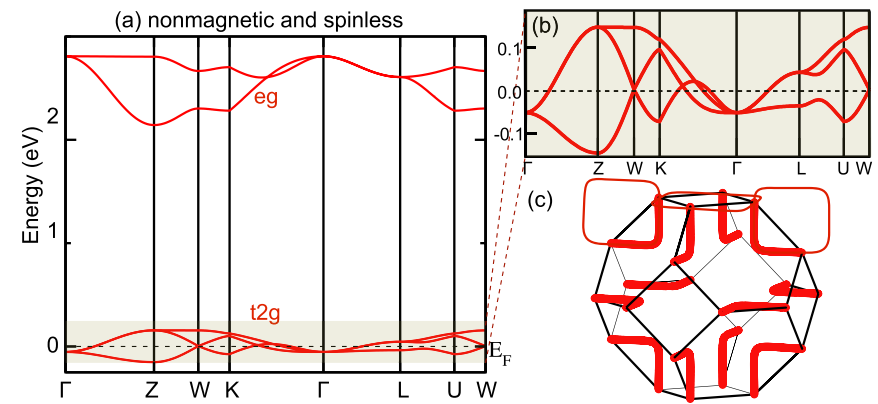

FIG. 2. Spinless electronic band structure and the nodal chain distribution of $\mathrm{Cs}_{2} \mathrm{MoCl}_{6}$ in the NM phase. (a) is the spinless electronic band structure of $\mathrm{Cs}_{2} \mathrm{MoCl}_{6}$ in the NM phase, where the $t_{2 g}$ orbits are magnified in (b). (c) shows the nodal chain band crossing formed by the lowest two bands of $t_{2 g}$ orbits.
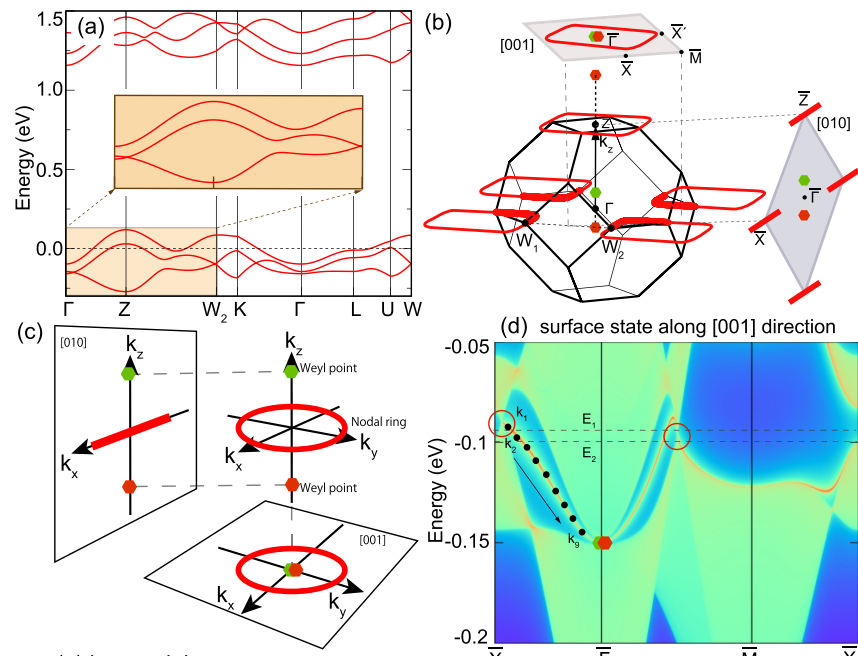

(d) surface state along [001] direction
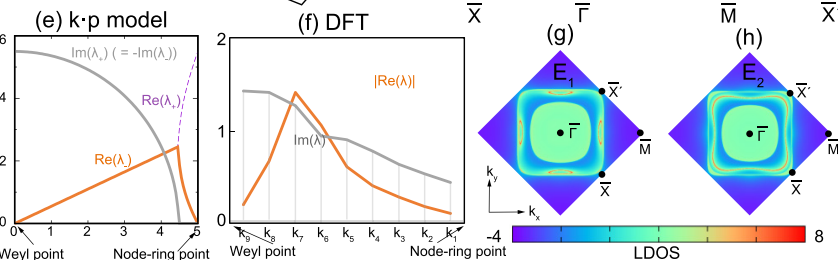

FIG. 3. Spinful electronic band structure and topological calculations on $\mathrm{Cs}_{2} \mathrm{MoCl}_{6}$ in the $\mathrm{FM}$ phase. (a) is the spinful electronic band structure of $\mathrm{Cs}_{2} \mathrm{MoCl}_{6}$ in the FM phase with a magnetic moment along the [001] direction. (b) shows the positions of a pair of Weyl points (red and green hexagons) and nodal rings in the bulk, (001), and (010) surface BZ. (c) Schematic figure of the projected positions of the nodal ring and Weyl points on the (001) and (010) surfaces. (d) shows the surface states, together with the projection of the Weyl points (red and green hexagons) and the nodal ring (red circles) on the (001) surface. (e) shows the real and imaginary parts for $\lambda_{+}$obtained by the $k \cdot p$ model with parameters of $\tilde{D}=1$ and $\sqrt{m}=5$, where $\operatorname{Im}\left(\lambda_{-}\right)=-\operatorname{Im}\left(\lambda_{+}\right)$. (f) is the absolute value of the real part $|\operatorname{Re}(\lambda)|$ and the imaginary part $\operatorname{Im}(\lambda)$ obtained by the DFT calculation, where the $k$ points are labeled in (d). (g) and (h) are the DSSs with different energies labeled in (d), where $E_{1}$ corresponds to the energy of nodal rings.

all the nodal rings will be pinned on the $M_{x}, M_{y}$, and $M_{z}$ planes, and meet with each other at the high-symmetry point $W$, forming a nodal chain semimetal, as shown in Figs. 2(b) and $2(\mathrm{c})$.

Figure 3(a) show the spinful electronic band structure of $\mathrm{Cs}_{2} \mathrm{MoCl}_{6}$ with the FM phase by the DFT $+U$ method [27], and the Hubbard $U$ term for Mo is $0.33 \mathrm{eV}$ obtained by the linear response method [28]. After considering the FM structure with a magnetic moment on the (001) surface and SOC, $M_{z}$ and $C_{4 z}$ will still be preserved, while $M_{x, y}$ and $C_{4 x, 4 y}$ will be broken. Thus, the nodal chain on the $M_{x, y}$ plane will open a gap but still leave a single nodal ring protected by the $M_{z}$ symmetry. However, the nodal ring will move slightly away from the $W$ point due to the symmetry breaking, and cross the high-symmetry line $W_{1}-W_{2}$ as shown in Fig. 3(b). Furthermore, SOC will make a change on the band structure along the $\Gamma-Z$ direction, and generate a pair of Weyl points protected by $C_{4 z}$ symmetry, as shown by the red and green hexagons in Fig. 3(b). Therefore, the $t_{2 g}$ bands will be 
significantly affected after considering the FM order and SOC, which make the nodal chains into a pair of Weyl points and a single nodal ring, as shown in Fig. 3(b).

$k \cdot p$ model analysis. Since two bands forming the Weyl points and a nodal ring are well separated from other bands, an effective $2 \times 2$ Hamiltonian based on the basis set $\left\{d_{x z}, d_{y z}\right\}$ can be constructed to describe the topological and other physical properties of the system. As discussed above, SOC and FM order will break the spin-rotation symmetry, $M_{x, y}$ and $C_{4 x, 4 y}$ of the system, reducing the symmetry of the system to $C_{4 h}$. Furthermore, the eigenvalues of the $\hat{C_{4 z}}$ operator for those two bands along the $\Gamma-Z$ direction are $e^{-\frac{\pi i}{4}}$ and $e^{\frac{-3 \pi i}{4}}$, so the $k \cdot p$ Hamiltonian describing the system around $Z$ with $C_{4 h}$ double group symmetry can be written to the lowest order in $\mathbf{k}$ as

$$
H\left(k_{x}, k_{y}, k_{z}\right)=\left(\begin{array}{cc}
m-|\boldsymbol{k}|^{2} & \tilde{D} k_{z} k_{+} \\
\tilde{D} k_{z} k_{-} & -m+|\boldsymbol{k}|^{2}
\end{array}\right)
$$

where $k_{ \pm}=k_{x} \pm i k_{y}$ and $\mathbf{k}$ is a wave vector measured from the $Z$ point. $\tilde{D}$ and $m$ are positive constants. We derived this Hamiltonian from the irreducible representations of the two bands around $Z$ after proper rescaling of the wave vector (see the Supplemental Material for details [29]). The eigenvalues of $H$ are $E= \pm \sqrt{\left(m-|\boldsymbol{k}|^{2}\right)^{2}+\tilde{D}^{2} k_{z}^{2}\left(k_{x}^{2}+k_{y}^{2}\right)}$, which form a pair of Weyl points and a single nodal ring located at $\left(k_{x}=0, k_{y}=0, k_{z}= \pm \sqrt{m}\right)$ and $\left(k_{x}^{2}+k_{y}^{2}=m, k_{z}=0\right)$ with $E=0$, respectively, as shown in Fig. 3(c).

As you may notice, the projections of topological band crossings on different surfaces will have different configurations. Namely, the two Weyl points will be projected inside of the nodal ring on the (001) surface, and to different sides of the projection of the nodal ring on the (010) surface [Fig. 3(c)]. This difference will lead to different types of surface-state connections, which will be explored by both DFT calculation and model analysis in the following. Topological surface states obtained by DFT are calculated by the Green's function method [30,31] with the tight-binding Hamiltonian obtained from maximally localized Wannier functions $[32,33]$.

Surface states of $F M \mathrm{Cs}_{2} \mathrm{MoCl}_{6}$ on the (001) surface. Figure 3(d) shows the surface states on the (001) surface, where two Weyl points will be projected onto the center of the nodal ring, i.e., $\bar{\Gamma}$, as shown in Fig. 3(b). It is worth mentioning that the topological surface states as well as the bulk bands are fully spin polarized in $\mathrm{Cs}_{2} \mathrm{MoCl}_{6}$. In order to get a better understanding of the surface-state connection and configuration, we also calculate the Fermi arcs with different energies, as shown in Figs. 3(g) and 3(h). The calculations show that the surface states on the (001) surface are in a drumhead shape, which is a characteristic of the nodal ring semimetal, and they will cross the projection of the Weyl points for systems with a coexistence of Weyl points and a nodal ring. Thus, we can control the density of the surface states or the Fermi velocity of the topological surface states on the (001) surface by modulating the energy of Weyl points and nodal rings. In the following, we will also use the analytical model for a further discussion on the universal connection pattern between a pair of Weyl points and a nodal ring.
According to the $k \cdot p$ model in Eq. (1), the nodal ring will be projected onto a circle $k_{x}^{2}+k_{y}^{2}=m$ and two Weyl points will be projected onto the $k_{x}=k_{y}=0$ point on the $(001)$ surface. In order to obtain the eigenvalues and eigenvectors of the surface states, we will deal with a semi-infinite system where the bulk and the vacuum lie in $z \leqslant 0$ and in $z>0$, respectively. In this case, $k_{z}$ is no longer a good quantum number, so we need to replace $k_{z}$ by $-i \partial_{z}[34,35]$ and obtain the Hamiltonian of the semi-infinite system,

$$
H_{z}\left(k_{x}, k_{y}\right)=\left(\begin{array}{cc}
m-R^{2}+\frac{\partial^{2}}{\partial z^{2}} & -i \tilde{D} R e^{i \theta} \frac{\partial}{\partial z} \\
-i \tilde{D} R e^{-i \theta} \frac{\partial}{\partial z} & -m+R^{2}-\frac{\partial^{2}}{\partial z^{2}}
\end{array}\right),
$$

where $k_{ \pm}=R e^{ \pm i \theta}$ with $R(>0)$ and $\theta$ being real. By introducing a trial wave function proportional to $e^{\lambda z}$ and a parameter $\lambda$ satisfying $\operatorname{Re}(\lambda)>0$ to represent a surface state, we obtain the corresponding eigenvectors

$$
\psi_{ \pm}(z)=\left(\begin{array}{c}
i \tilde{D} R e^{i \theta} \lambda_{ \pm} \\
m-R^{2}+\lambda_{ \pm}^{2}-E
\end{array}\right) e^{\lambda_{ \pm} z}
$$

where $\lambda_{ \pm}^{2}=\frac{1}{2} \tilde{D}^{2} R^{2}-\left(m-R^{2}\right) \pm \sqrt{E^{2}-\left(m-R^{2}\right) \tilde{D}^{2} R^{2}+\frac{1}{4} \tilde{D}^{4} R^{4}}$. If we impose a boundary condition $\psi(z=0)=0$ for an eigenvector of the form $\psi=C_{+} \psi_{+}+C_{-} \psi_{-}\left(C_{ \pm}\right.$: constant $)$, we obtain dispersionless DSSs with $E=0$, and $\lambda_{ \pm}$becomes $\lambda_{ \pm}=\frac{\tilde{D} R}{2} \pm \sqrt{\left(\frac{\tilde{D} R}{2}\right)^{2}+R^{2}-m}$ (see the Supplemental Material for details [29]). In order to meet the condition of $\operatorname{Re}(\lambda)>$ $0, R$ should satisfy $|R| \leqslant \sqrt{m}$. Therefore, the DSSs cross the projection of the Weyl points lying at $E=0$, which match with our DFT calculations very well.

Next, we will study the penetration length for the surface states on the (001) surface, which have a unique connection between Weyl points and the nodal ring, by both the analytical model and DFT calculation separately. The penetration length $l$ for a wave function proportional to $e^{\lambda z}\left(\lambda=\lambda_{ \pm}\right)$is given by $l_{ \pm}=\frac{1}{\operatorname{Re}\left(\lambda_{ \pm}\right)}$. Here, we consider $l_{-}$as the penetration depth because $l_{-} \geqslant l_{+}$. When the surface state approaches the Weyl points or the nodal ring, the penetration length $l_{-}$ is expected to be divergent due to the bulk nature of these bands. Figure 3(e) shows the values of $\lambda_{ \pm}$as a function of $R$, which is between the projection of the Weyl points $(R=0)$ and the nodal ring $(R=\sqrt{m})$. We notice that $R=\tilde{R}=\sqrt{\frac{4 m}{\tilde{D}^{2}+4}}$ is a special point in that $\lambda_{+}=\lambda_{-}$is satisfied and the behavior of $l_{ \pm}$changes drastically. When $R>\tilde{R}, \lambda_{ \pm}$are real and the surface state decays into the bulk without oscillation. On the other hand, when $R<\tilde{R}, \lambda_{ \pm}$are complex and the surface states show an oscillating decay into the bulk. Further calculations obtained by a 47-layer slab with the tight-binding Hamiltonian obtained from maximally localized Wannier functions also show the same trend with the model calculation for both the real and imaginary parts of $\lambda$, which are shown in Fig. 3(f).

Surface states of $F M \mathrm{Cs}_{2} \mathrm{MoCl}_{6}$ on the (010) surface. On the (010) surface, two Weyl points will be projected on the $\bar{\Gamma}-\bar{Z}$ high-symmetry line, while the nodal ring will be projected to a line near $\bar{X}$ and $\bar{Z}$, as shown in Figs. 3(b) and 3(c). Figures 4(b)-4(d) show the Fermi arcs with different energies labeled in Fig. 4(a), where $E_{2}$ corresponds to the energy of the 

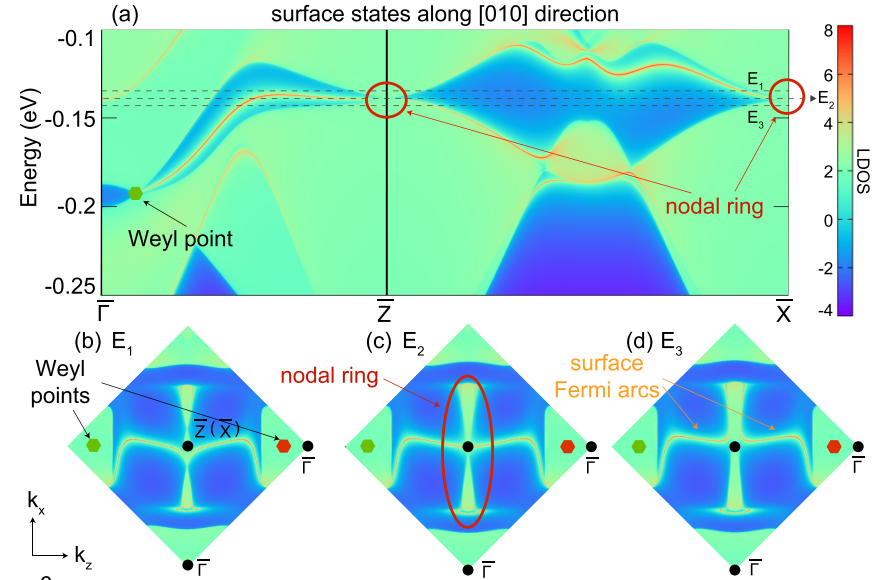

(c) $\mathrm{E}_{2}$

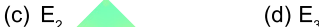

(d)
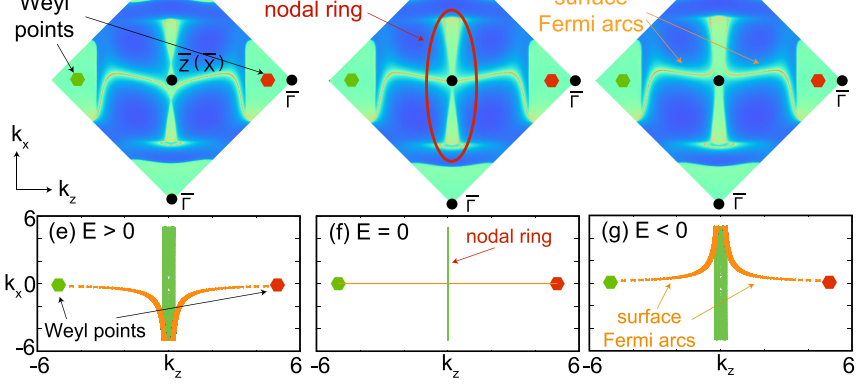

FIG. 4. Surface-state calculation on the (010) surface for $\mathrm{Cs}_{2} \mathrm{MoCl}_{6}$. (a) is the surface state on the (010) surface. (b)-(d) are the Fermi arcs with different energies labeled in (a), where $E_{2}$ corresponds to the energy of the nodal ring projected at $\bar{Z}$. (e) $-(\mathrm{g})$ are Fermi-arc calculations by the $k \cdot p$ model with $E>0, E=0$, and $E<0$. Parameters are $\tilde{D}=1$ and $\sqrt{m}=5$. The red and green hexagons represent the Weyl nodes with chiralities +1 (monopole) and -1 (antimonopole), respectively.

nodal ring projected at $\bar{Z}$. When the energy for the isoenergetic contour decreases from $E_{1}$ to $E_{3}$, the Fermi arcs from the Weyl points will cross through the projected points of the nodal ring, and rotate around two Weyl points. Thus, surface states on the (010) surface are in a helicoid shape and show the feature of Weyl points, which is different with the surface states on the (001) surface showing the feature of a nodal ring.

These Fermi-arc surface states agree with expectations from bulk quantities. At the $\Gamma$ point $\left(k_{z}=0\right)$, the $\Gamma_{6}^{+}\left(C_{4}=\right.$ $\left.e^{-\frac{\pi i}{4}}\right)$ irreducible representation has a lower energy than $\Gamma_{7}^{+}$ $\left(C_{4}=e^{-\frac{3 \pi i}{4}}\right)$, and at the $Z$ point $\left(k_{z}=\pi\right)$, the $Z_{7}^{+}\left(C_{4}=\right.$ $\left.e^{-\frac{3 \pi i}{4}}\right)$ irreducible representation has a lower energy than $Z_{6}^{+}\left(C_{4}=e^{-\frac{\pi i}{4}}\right)$. From this band inversion between different $C_{4}$ eigenstates, we can derive the difference of Chern numbers [ $\left.\mathrm{Ch}\left(k_{z}\right)\right]$ between the $k_{z}=0$ and $k_{z}=\pi$ subspaces. From Refs. [36,37], we get $\frac{i^{\operatorname{chh}\left(k_{z}=\pi-\delta\right)}}{i^{\mathrm{ch}\left(k_{z}=\delta\right)}}=\frac{e^{-\frac{3 \pi i}{4}}}{e^{-\frac{\pi i}{4}}}=-i$. Namely, $\mathrm{Ch}\left(k_{z}=\pi-\delta\right)-\mathrm{Ch}\left(k_{z}=\delta\right) \equiv-1(\bmod 4)$. Here, $\delta$ is a small constant, which is introduced to make the spectrum gapped by avoiding the nodal ring. This difference of Chern numbers is caused by the Weyl nodes at $\mathbf{k}=\left(0,0, \pm k_{0}\right)$ $\left(k_{0}>0\right)$, with a monopole charge $\mp 1$, respectively. In the present case, $\mathrm{Ch}\left(k_{z}\right)=0$ for $0<\left|k_{z}\right|<k_{0}$ and $\mathrm{Ch}\left(k_{z}\right)=-1$ for $k_{0}<\left|k_{z}\right|<\pi$, leading to topological chiral surface states with a negative velocity $v_{x}$ on the (010) surface. It perfectly agrees with Figs. 4(b)-4(d).

Further model calculations on the (010) surface also verify this point, as shown in Figs. 4(e)-4(g) (see the Supplemental Material for details [29]). The projection of the Weyl points and the nodal ring are located at $\left(k_{x}, k_{z}\right)=(0, \pm \sqrt{m})$ and $\left(k_{z}=0,-\sqrt{m} \leqslant k_{x} \leqslant \sqrt{m}\right)$, respectively. When $E=0$ [Fig. 4(f)], the Fermi arcs cross the projected nodal ring and connect the two Weyl points straightly; when $E>0$ and $E<0$ [Figs. 4(f) and 4(g)], the Fermi arcs rotate around two Weyl points and cross through the nodal rings. Remarkably, when $E \neq 0$, the Fermi arc meets the nodal ring tangentially, and the direction of the Fermi arc near the nodal ring changes abruptly across $E=0$, as shown in Figs. 4(e)-4(g). It is due to an interplay between the helicoid nature of the surface state and the tangential property between the surface Fermi arc and a bulk Fermi surface. This abrupt change of the Fermi arc also appears in Figs. 4(b)-4(d) from the DFT. Both the DFT calculation and the model calculation on the Fermi arcs show a perfect match with each other, which proves the correctness of our theory on the unique surface-state connection.

We notice that such a unique surface-state connection behavior can be found in other materials with different symmetries, such as in the electronic band structure of FM $\mathrm{HgCr}_{2} \mathrm{Se}_{4}$ (with SOC and without $\mathcal{T}$ ) [6], the electronic band structure of FM double perovskites $\left(\mathrm{Ba}_{2} \mathrm{NaOsO}_{6}, \mathrm{Sr}_{2} \mathrm{SrOsO}_{6}\right.$, $\mathrm{Ba}_{2} \mathrm{ZnReO}_{6}, \mathrm{Ba}_{2} \mathrm{MgReO}_{6}$, and $\mathrm{Ba}_{2} \mathrm{CdReO}_{6}$, with $\mathrm{SOC}$ and without $\mathcal{T}$ ) [22,23], and the phonon band structure of oxide perovskites $\left(\mathrm{BaTiO}_{3}, \mathrm{PbTiO}_{3}\right.$, without $\mathrm{SOC}$ and with $\left.\mathcal{T}\right)$ [25]. However, none of those works discuss the connection of the unique surface states, and they mainly focus on the topology of the bulk bands. We demonstrate that both the drumhead surface states and the helicoid surface states will cross the projected points of the Weyl and nodal ring along different directions. Such a surface-state connection for systems with a coexistence of Weyl points and nodal rings (and nodal lines) is universal, and it is also applicable to systems with or without considering SOC and $\mathcal{T}$.

Conclusion. We propose a FM topological half metal $\mathrm{Cs}_{2} \mathrm{MoCl}_{6}$ with spin-polarized bulk bands and surface states near the Fermi level. Due to the coexistence of Weyl points and a nodal ring, the surface states of $\mathrm{Cs}_{2} \mathrm{MoCl}_{6}$ show different properties along different directions. On the (001) surface, the surface states are in the drumhead shape, and they always go across the Weyl nodes. On the (010) surface, the Fermi-arc surface states are in a helicoid shape, and they meet the nodal ring tangentially, with their shapes changing abruptly as a function of the energy. An analytical model calculation also matches with the DFT calculation on both surface-state connections and configurations, which helps to verify our theory on the topological surface states. Such a unique feature of the surface-state connection is universal for systems with a coexistence of Weyl points and nodal rings, irrespective of the presence or absence of time-reversal symmetry and spin-orbit coupling.

Acknowledgments. We acknowledge support from JSPS KAKENHI Grants No. JP18H03678 and No. JP20H04633, the Tokodai Institute for Element Strategy (TIES) funded by MEXT Elements Strategy Initiative to Form Core Research Center. T.Z. also acknowledges support by the Japan Society for the Promotion of Science (JSPS), KAKENHI Grant No. JP21K13865.

T.Z. and D.H. contributed equally to this work. 
[1] J. Zou, Z. He, and G. Xu, The study of magnetic topological semimetals by first principles calculations, npj Comput. Mater. 5, 1 (2019).

[2] S. Nie, G. Xu, F. B. Prinz, and S.-C. Zhang, Topological semimetal in honeycomb lattice LnSi, Proc. Natl. Acad. Sci. USA 114, 10596 (2017).

[3] R.-W. Zhang, Z. Zhang, C.-C. Liu, and Y. Yao, Nodal Line Spin-Gapless Semimetals and High-Quality Candidate Materials, Phys. Rev. Lett. 124, 016402 (2020).

[4] Z. Wang, M. G. Vergniory, S. Kushwaha, M. Hirschberger, E. V. Chulkov, A. Ernst, N. P. Ong, Robert J. Cava, and B. A. Bernevig, Time-Reversal-Breaking Weyl Fermions in Magnetic Heusler Alloys, Phys. Rev. Lett. 117, 236401 (2016).

[5] S. Nie, Y. Sun, F. B. Prinz, Z. Wang, H. Weng, Z. Fang, and X. Dai, Magnetic Semimetals and Quantized Anomalous Hall Effect in $\mathrm{EuB}_{6}$, Phys. Rev. Lett. 124, 076403 (2020).

[6] G. Xu, H. Weng, Z. Wang, X. Dai, and Z. Fang, Chern Semimetal and the Quantized Anomalous Hall Effect in $\mathrm{HgCr}_{2} \mathrm{Se}_{4}$, Phys. Rev. Lett. 107, 186806 (2011).

[7] J. Noky, Q. Xu, C. Felser, and Y. Sun, Large anomalous Hall and Nernst effects from nodal line symmetry breaking in $\mathrm{Fe}_{2} \mathrm{Mn} X$ ( $\left.X=\mathrm{P}, \mathrm{As}, \mathrm{Sb}\right)$, Phys. Rev. B 99, 165117 (2019).

[8] I. Belopolski, K. Manna, D. S. Sanchez, G. Chang, B. Ernst, J. Yin, S. S. Zhang, T. Cochran, N. Shumiya, H. Zheng et al., Discovery of topological Weyl fermion lines and drumhead surface states in a room temperature magnet, Science $\mathbf{3 6 5}, 1278$ (2019).

[9] C.-Z. Chang, J. Zhang, X. Feng, J. Shen, Z. Zhang, M. Guo, K. Li, Y. Ou, P. Wei, L.-L. Wang et al., Experimental observation of the quantum anomalous Hall effect in a magnetic topological insulator, Science 340, 167 (2013).

[10] K. Kuroda, T. Tomita, M.-T. Suzuki, C. Bareille, A. A. Nugroho, P. Goswami, M. Ochi, M. Ikhlas, M. Nakayama, S. Akebi et al., Evidence for magnetic Weyl fermions in a correlated metal, Nat. Mater. 16, 1090 (2017).

[11] K. Kim, J. Seo, E. Lee, K.-T. Ko, B. S. Kim, B. G. Jang, J. M. Ok, J. Lee, Y. J. Jo, W. Kang et al., Large anomalous Hall current induced by topological nodal lines in a ferromagnetic van der Waals semimetal, Nat. Mater. 17, 794 (2018).

[12] Q. Wang, Y. Xu, R. Lou, Z. Liu, M. Li, Y. Huang, D. Shen, H. Weng, S. Wang, and H. Lei, Large intrinsic anomalous Hall effect in half-metallic ferromagnet $\mathrm{Co}_{3} \mathrm{Sn}_{2} \mathrm{~S}_{2}$ with magnetic Weyl fermions, Nat. Commun. 9, 3681 (2018).

[13] P. Li, J. Koo, W. Ning, J. Li, L. Miao, L. Min, Y. Zhu, Y. Wang, N. Alem, C.-X. Liu et al., Giant room temperature anomalous Hall effect and tunable topology in a ferromagnetic topological semimetal $\mathrm{Co}_{2} \mathrm{MnAl}$, Nat. Commun. 11, 3476 (2020).

[14] B. W. Xia, Y. J. Jin, J. Z. Zhao, Z. J. Chen, B. B. Zheng, Y. J. Zhao, R. Wang, and H. Xu, Robust Twin Pairs of Weyl Fermions in Ferromagnetic Oxides, Phys. Rev. Lett. 122, 057205 (2019).

[15] N. Morali, R. Batabyal, P. K. Nag, E. Liu, Q. Xu, Y. Sun, B. Yan, C. Felser, N. Avraham, and H. Beidenkopf, Fermi-arc diversity on surface terminations of the magnetic Weyl semimetal $\mathrm{Co}_{3} \mathrm{Sn}_{2} \mathrm{~S}_{2}$, Science 365, 1286 (2019).

[16] D. F. Liu, A. J. Liang, E. K. Liu, Q. N. Xu, Y. W. Li, C. Chen, D. Pei, W. J. Shi, S. K. Mo, P. Dudin et al., Magnetic Weyl semimetal phase in a Kagomé crystal, Science 365, 1282 (2019).

[17] Y. Xu, L. Elcoro, Z.-D. Song, B. J. Wieder, M. G. Vergniory, N. Regnault, Y. Chen, C. Felser, and B. A. Bernevig, High- throughput calculations of magnetic topological materials, Nature (London) 586, 702 (2020).

[18] D. Zhang, M. Shi, T. Zhu, D. Xing, H. Zhang, and J. Wang, Topological Axion States in the Magnetic Insulator $\mathrm{MnBi}_{2} \mathrm{Te}_{4}$ with the Quantized Magnetoelectric Effect, Phys. Rev. Lett. 122, 206401 (2019).

[19] Y. Xu, Z. Song, Z. Wang, H. Weng, and X. Dai, Higher-Order Topology of the Axion Insulator EuIn $\mathrm{As}_{2}$, Phys. Rev. Lett. 122, 256402 (2019).

[20] J. Li, Y. Li, S. Du, Z. Wang, B.-L. Gu, S.-C. Zhang, K. He, Wenhui Duan, and Y. Xu, Intrinsic magnetic topological insulators in van der Waals layered $\mathrm{MnBi}_{2} \mathrm{Te}_{4}$-family materials, Sci. Adv. 5, eaaw5685 (2019).

[21] B. Hu, P. Wang, Y. Xiao, and L.-P. Song, Crystal structure of dicesium hexachloromolybdate (IV), $\mathrm{Cs}_{2}\left[\mathrm{MoCl}_{6}\right]$, Z. Kristallogr. 220, 318 (2005).

[22] Y.-J. Song and K.-W. Lee, Symmetry-protected spinful magnetic Weyl nodal loops and multi-Weyl nodes in $5 d^{n}$ cubic double perovskites $(n=1,2)$, Phys. Rev. B 102, 035155 (2020).

[23] X. Zhao, P.-J. Guo, F. Ma, and Z.-Y. Lu, Coexistence of topological Weyl and nodal-ring states in ferromagnetic and ferrimagnetic double perovskites, Phys. Rev. B 103, 085138 (2021).

[24] X.-Q. Sun, S.-C. Zhang, and T. Bzdušek, Conversion Rules for Weyl Points and Nodal Lines in Topological Media, Phys. Rev. Lett. 121, 106402 (2018).

[25] B. Peng, Y. Hu, S. Murakami, T. Zhang, and B. Monserrat, Topological phonons in oxide perovskites controlled by light, Sci. Adv. 6, eabd1618 (2020).

[26] J. W. Quail and G. A. Rivett, Complex fluorides of tetravalent cobalt, Can. J. Chem. 50, 2447 (1972).

[27] S. L. Dudarev, G. A. Botton, S. Y. Savrasov, C. J. Humphreys, and A. P. Sutton, Electron-energy-loss spectra and the structural stability of nickel oxide: An LSDA+U study, Phys. Rev. B 57, 1505 (1998).

[28] M. Cococcioni and S. de Gironcoli, Linear response approach to the calculation of the effective interaction parameters in the LDA + U method, Phys. Rev. B 71, 035105 (2005).

[29] See Supplemental Material at http://link.aps.org/supplemental/ 10.1103/PhysRevResearch.3.L042037 for (i) density of states for nonmagnetic $\mathrm{Cs}_{2} \mathrm{MoCl}_{6}$, (ii) electronic band structure for $\mathrm{Cs}_{2} \mathrm{MoCl}_{6}$ with different magnetic orders, (iii) derivation of the effective model, (iv) surface-state calculations on the (001) surface by the $k \cdot p$ model, and (v) surface-state calculations on the (010) surface by the $k \cdot p$ model.

[30] M. P. Lopez Sancho, J. M. Lopez Sancho, and J. Rubio, Quick iterative scheme for the calculation of transfer matrices: Application to Mo (100), J. Phys. F: Met. Phys. 14, 1205 (1984).

[31] Q. S. Wu, S. N. Zhang, H.-F. Song, M. Troyer, and A. A. Soluyanov, WannierTools: An open-source software package for novel topological materials, Comput. Phys. Commun. 224, 405 (2018).

[32] A. A. Mostofi, J. R. Yates, Y.-S. Lee, I. Souza, D. Vanderbilt, and N. Marzari, wannier90: A tool for obtaining maximallylocalised Wannier functions, Comput. Phys. Commun. 178, 685 (2008).

[33] N. Marzari, A. A. Mostofi, J. R. Yates, I. Souza, and D. Vanderbilt, Maximally localized Wannier functions: Theory and applications, Rev. Mod. Phys. 84, 1419 (2012). 
[34] B. Zhou, H.-Z. Lu, R.-L. Chu, S.-Q. Shen, and Q. Niu, Finite Size Effects on Helical Edge States in a Quantum Spin-Hall System, Phys. Rev. Lett. 101, 246807 (2008).

[35] M. Wada, S. Murakami, F. Freimuth, and G. Bihlmayer, Localized edge states in two-dimensional topological insulators: Ultrathin Bi films, Phys. Rev. B 83, 121310(R) (2011).
[36] C. Fang, M. J. Gilbert, X. Dai, and B. A. Bernevig, Multi-Weyl Topological Semimetals Stabilized by Point Group Symmetry, Phys. Rev. Lett. 108, 266802 (2012).

[37] C. Fang, M. J. Gilbert, and B. A. Bernevig, Bulk topological invariants in noninteracting point group symmetric insulators, Phys. Rev. B 86, 115112 (2012). 